Original article

\title{
Spatio-temporal pattern of malignant neoplasm of brain in Fars province I.R.Iran (2011-2015): A population-based cross sectional study
}

\author{
Abbas Rezaianzadeh $^{\mathrm{a}}$, Fatemeh Jafari ${ }^{\mathrm{b}, * *}$, Zahra Khosravizadegan ${ }^{\mathrm{c}}$, Marzieh Eslahi ${ }^{\mathrm{d}}$

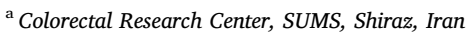 \\ ${ }^{\mathrm{b}}$ MSc Candidate of Epidemiology in Shiraz University of Medical Science, Shiraz, Iran \\ ${ }^{\mathrm{c}}$ Fars Cancer Registry, SUMS, Shiraz, Iran \\ ${ }^{\mathrm{d}}$ Department of Biostatistics and Epidemiology, School of Health, Kerman University of Medical Science, Kerman, Iran
}

A R T I C L E I N F O

\section{Keywords:}

Incidence

Neoplasms

Brain

Registries

Iran

\begin{abstract}
A B S T R A C T
Background: Tumors of the central nervous system (CNS) are a heterogeneous group of neoplasms with different prevalence in different genders, age groups and various parts of the CNS. This study aimed to investigate the incidence of malignant neoplasm of brain (C71) based on Fars population data.

Methods: This study was cross-sectional, carried out based on Fars population -based Cancer Registry (FPCR) data. Data was collected retrospectively reviewing all malignant neoplasm of brain patients' records during a 5year period (2011-2015). Age Standardized Incidence Rates (ASR) were calculated based on world population standards. To analyze the ASR time trends by gender, we carried out a chi-square goodness of fit test.

Results: Findings revealed that 1043 cases of malignant neoplasm of brain were registered, 438 (\%41.9) of which were women. Malignant neoplasm of brain became the eighth malignancy in 2015 in both genders. Its incidence was higher in men (26.15/100000) than in women (19.45/100 000). There was an increasing but not significant trend from 2011 to 2013 in both men and women ( $\mathrm{p}=0.722, \mathrm{p}=0.478$, respectively). The highest increase was in 2013 (ASR $=6.64$ in men and ASR $=5.71$ in women per 100,000).

Conclusion: Brain and other CNS tumors are an important source of cancer deaths around the world. Regional variations in incidence may provide clues leading to genetic and/or environmental causes as well as a basis for expanding knowledge about their epidemiology. This study demonstrated that the trend had been increasing in the years during which the study was conducted.
\end{abstract}

\section{Introduction}

Central nervous system (CNS) tumors are a diverse group of benign and malignant tumors arising from the brain parenchyma and its surrounding structures. ${ }^{1}$ These tumors are the leading cause of cancer-related mortality in children and also the most common solid tumors that occur in childhood, they account for $20 \%$ of all neoplasms. ${ }^{2}$ Brain tumors make up about $88 \%$ of all CNS tumors. ${ }^{3}$ The factor that may cause brain tumors is uncertain. White race, exposure to radiation, certain chemical exposures in the workplace, working in the rubber, petrochemical or metal industries and family history of malignant neoplasm of brain are known to increase the risk of brain and other CNS tumors. ${ }^{4}$ Although, CNS tumors account for about $3 \%$ of the new cancer diagnoses globally, they can lead to mortality and morbidity that often creates a high burden on both families and health care systems. ${ }^{1,5}$ The incidence of primary CNS tumors has been estimated as 3.9 and 3.2 per
100,000 person-year worldwide in males and females, respectively. ${ }^{6}$ The increase observed in incidence rates of malignant brain and other CNS tumors over the past decades is mainly due to increased access to diagnostic imaging and specialized medical care. ${ }^{7}$ In addition, their distribution varies throughout the world, with the highest incidence in Australia, North America and European countries (men, 5.8 per 100,000; women, 4.1 per 100,000), especially among the white people, and the lowest in Africa (men, 3.0 per 100,000; women, 2.1 per 100,000). ${ }^{8,9}$ Epidemiological studies in different parts of the world have shown that males have a higher risk of being diagnosed with malignant brain and other CNS tumors than females. ${ }^{10}$ It is estimated that about 50,800 new cancer cases occur in Iran each year. Among them more than $53 \%$ belong to males. Almost $4 \%$ of all deaths due to all cancer cases in both genders in this country are due to malignant brain tumors. ${ }^{11,12}$ Due to the lack of population based research on the epidemiology of malignant neoplasm of brain in Iran, this study was

\footnotetext{
* Corresponding author. Mobile: + 989178149262.

E-mail address: m.jafarii4319@gmail.com (F. Jafari).
} 


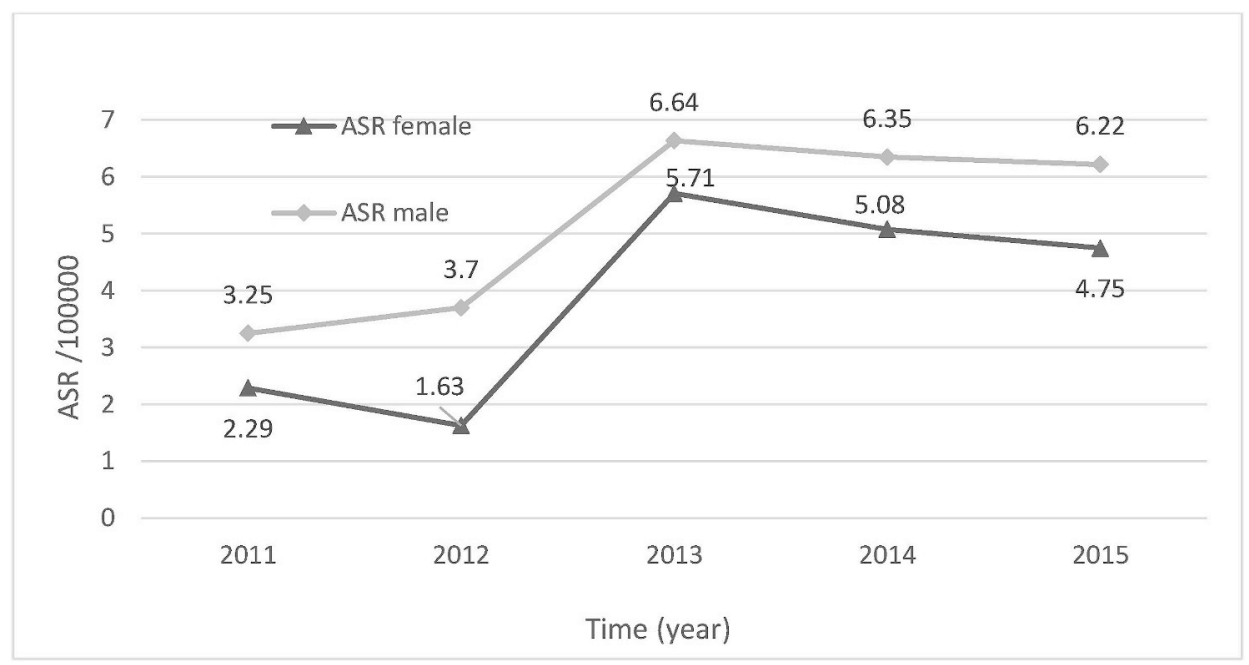

Fig. 1. Trends of Age Standardized Incidence Rates (ASR) by genders in Fars province in 2011-2015.

conducted to evaluate the spatio-temporal pattern of this neoplasm. The comparison of this data with national and global statistics and trends will provide the basis for better planning in different areas of health and development, including evaluating the effectiveness of the regional health care system in Fars province.

\section{Study location}

Fars Province is located in the southwest of Iran. It has an area of 122,608 square kilometers. According to the latest census in 2016, Fars had a population of more than 4.8 million and is the fourth largest province in Iran, it also has 29 counties, 83 sections and 204 rural districts.

\section{Methods}

This is a cross-sectional study, carried out based on Fars populationbased Cancer Registry (FPCR) data. Data was collected retrospectively reviewing all malignant neoplasm of brain patient records during a 5year period (2011-2015). This data was derived from reports of centers which provide diagnostic and therapeutic services like hospitals, clinics, pathology labs, radiology clinics, radiotherapy clinics, imaging centers in Shiraz (capital of Fars province) and other cities. A limited portion of the information was received as paper based from some centers in Shiraz and another cities. The total population and subcategories according to gender and age groups were taken from the census of the Statistical Center of Iran, then the crude incidence rate and the age standardized incidence rate (ASR) per 100,000 persons were calculated. We used the direct standardized method using world standard population. ${ }^{13}$ The statistical population included all patients diagnosed with malignant neoplasm of brain. The information comprised of diagnosis date, age, gender, primary site codes, morphology codes (according to ICD-O-3, C71) and the residence locations of the patients. In the rechecking process for details of the entered data, duplicates were removed in three stages. The first step was for items that had been registered in several sources, one of which should be saved. In the second step, an expert evaluated primary and secondary or metastatic cases and compared them with reports of at least the past three years and the first report was saved. The third step was related to the uncertain items which were consulted with an expert pathologist. Finally, to describe the incidence time trends, we carried out a chi-square goodness of fit test by gender. At this point, firstly ASR was calculated by dividing the number of disease cases by the population of each year, in men and women separately. The ASR was then multiplied by the standard population to achieve the expected frequency. For examining the significance of the time trend of the cancer, the non-parametric chisquare test was used after weighing the sum of the expected amount. Additionally, ArcGIS software version 10.4.1 Product by American company ESRI was used to display the geographical distribution of CNS tumors.

\section{Results}

Findings revealed that 1043 patient were registered 438 (\%41.9) of which were women. The ASR was higher in men (26.16/100000) than in women (19.46/100 000). Overall, there was an increasing but not significant trend from 2011 to 2013 in both men and women ( $\mathrm{p}=0.722, \mathrm{p}=0.478$, respectively). The highest increase was in 2013 (ASR $=6.64$ in men and ASR $=5.71$ in women per 100,000). The ASR increased from 2.29 to 4.75 in women and from 3.25 to 6.22 in men during 2011-2015 (Fig. 1). The incidence of cancer in cities like Eghlid, Arsanjan, Firoozabad, Sarvestan, Fasa, Jahrom, Lamerd, Darab and Lar was incremental and in Ghaderabad, Farashband, Ghirokarzin, Khonj, Pasargad it was decreasing. Arsanjan, Lamerd, Eghlid and Sepidan had the most frequency in 2015 (Figs. 2 and 3).

All in all, the ASR trend showed a growth until 2012 and then it was leveled off in both genders. The peak of increase was seen in 2012 (Figs. 2 and 3). Malignant neoplasm of brain has become more common by time. For example, it was known respectively as the seventh and eighth cancer in 2014 and 2015 in both genders (Table 1).

Fig. 4 shows age distribution of malignant neoplasm of brain with some fluctuations in different age groups. The highest increase in women is in the age groups of 25-29 years and in men 30-39 and 60-64 years.

\section{Discussion}

Malignant neoplasm of brain though not frequent, contributes significantly to morbidity because of its relatively poor survival rate. ${ }^{4}$ The incidence of CNS tumors in Iran is in accordance with the incidence of CNS tumors in other developing countries. Incidence of 2.74 malignant and 2.95 benign lesions per 100,000 person-years and a total of 5.69 per 100,000 person-years for primary brain tumors in Iran. ${ }^{14}$

According to the results of this study, men with malignant neoplasm of brain have a greater abundance. Miranda-Filho and colleagues observed that men had on average a $40 \%$ higher incidence of brain and 


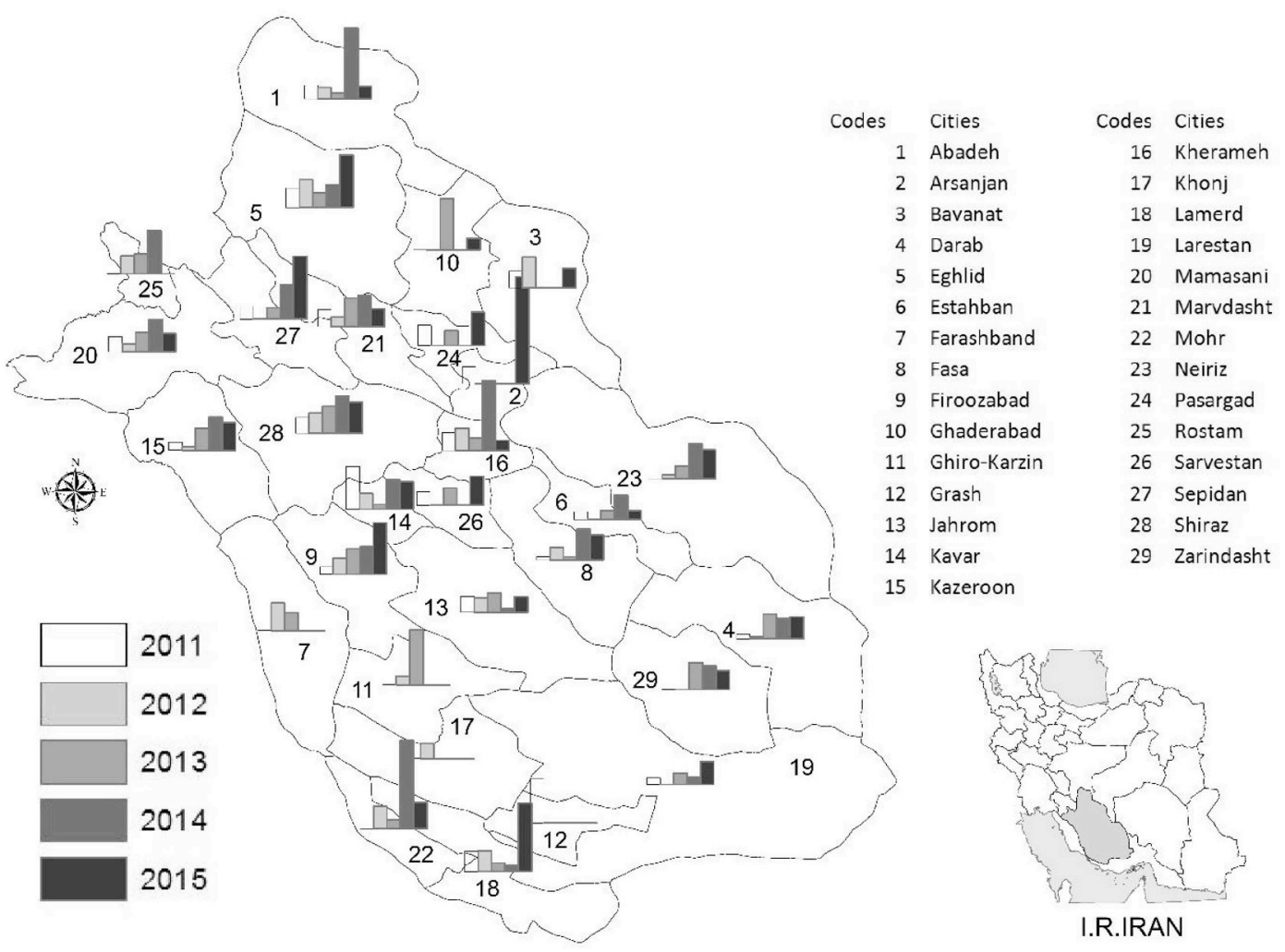

Fig. 2. Spatio-temporal pattern of malignant neoplasm of brain in male and different cities of Fars province during 2011-2015.

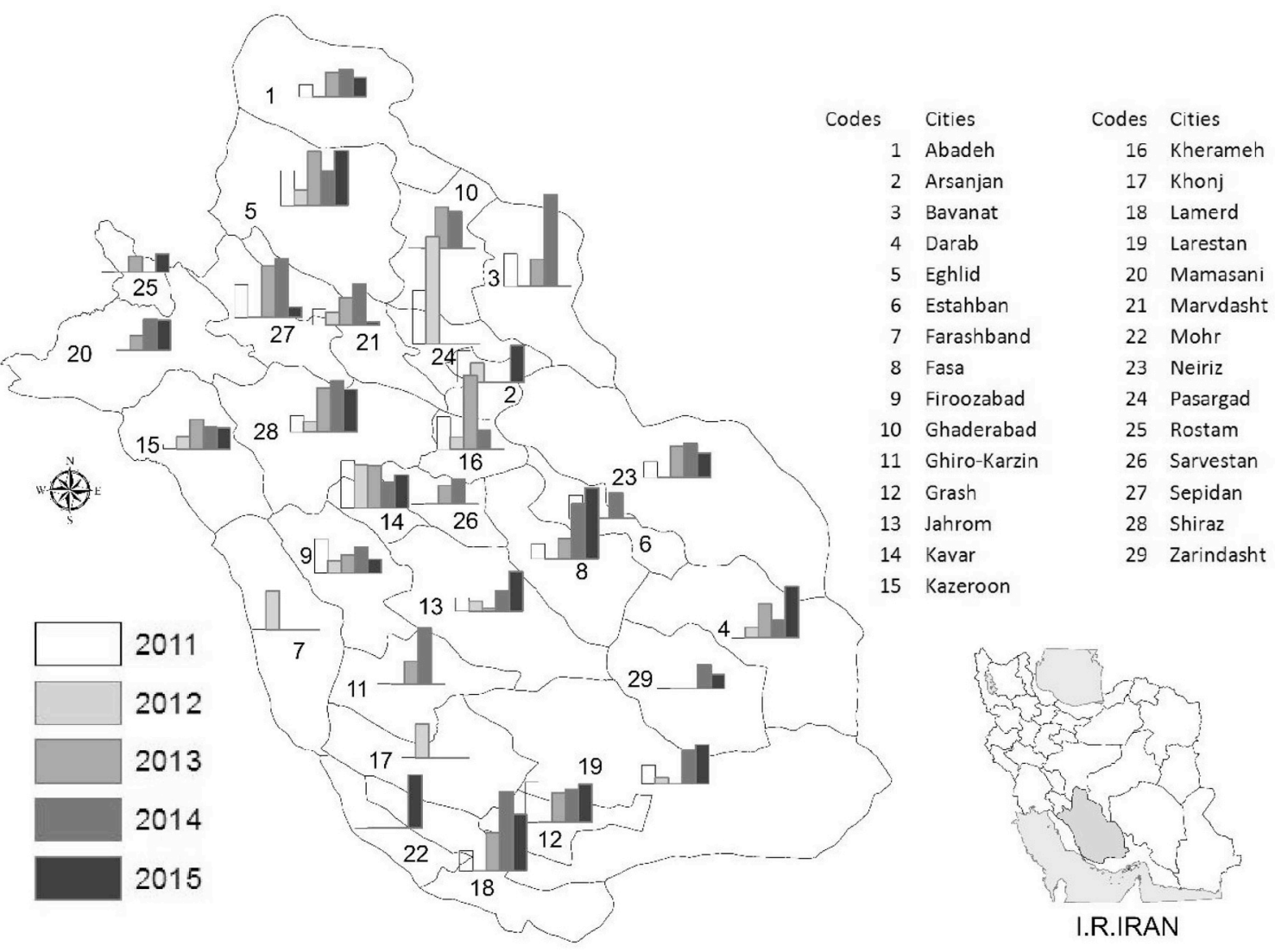

Fig. 3. Spatio-temporal pattern of malignant neoplasm of brain in female and different cities of Fars province during 2011-2015. 
Table 1

Order of malignant neoplasm of brain by gender in Fars province in 2011-2015.

\begin{tabular}{lllllll}
\hline Year & & 2011 & 2012 & 2013 & 2014 & 2015 \\
\hline $\begin{array}{c}\text { Order of cancer except non- } \\
\text { melanoma skin cancer }\end{array}$ & Men & 10 & 11 & 7 & 7 & 8 \\
& Women & 13 & 17 & 5 & 7 & 8 \\
\hline
\end{tabular}

other CNS cancers than women. ${ }^{15}$ It has been suggested that gender differences related to the genders' hormones and genetic features may be causative factors. ${ }^{16}$ In addition, adverse working conditions, including a higher exposure to pesticides, chemicals, and biological agents, among men may be a contributing factor. ${ }^{17}$ However, the incidence of primary brain tumors in the US, ${ }^{18}$ Korea $^{19}$ and France ${ }^{20}$ is more frequent in females. On the other hand, Sean concluded that there was no significant difference in the incidence of this cancer between the two genders. ${ }^{21}$

The current study reveals an increase in incidence during the study period. A study done by Pirouzmand et al. found an increase in the incidence of this cancer that is consistent with the results of this study. ${ }^{22}$ Other possible explanations are attributable to improvements in diagnostic imaging, increased availability of medical care and neurosurgeons, changing approaches in the treatment of older patients and changes in classifications of specific histology of brain tumors. ${ }^{7,23,24}$ In Japan, a decreasing incidence has been reported over the period 1995-2004 and for the whole country, a decreasing trend has been observed since $1987 .{ }^{25}$

In this survey, age distribution has no particular trend but the highest increase in women was in the age group of 25-29 years and for men it was in the 30-39 and 60-64 age groups. In the study of Monaharan et al. the majority of the tumors occurred in the age group of $45-54$ both in males and females. ${ }^{4}$ Other studies have demonstrated a higher prevalence of CNS tumors in older populations. ${ }^{26-29}$ The increased incidence in the elderly has been attributed to a possible reduction in immunological surveillance or an increased number of somatic mutations that occur over a lifetime. ${ }^{30}$

As mentioned, the incidence of cancer in some cities like Eghlid, Arsanjan, Firoozabad, Sarvestan, Fasa, Jahrom, Lamerd, Darab and Lar was incremental. In 2015, the highest rates were in Arsanjan and Fasa, respectively, in men and women. The results may be related to improved accessibility of advanced neuroimaging facilities and better CNS tumor registration practices. ${ }^{31}$ The evaluation of brain cancer was performed in Fars province for the first time. For this reason, there is no information about the geographical trend in different parts of the province making it difficult to leave any comments on this matter.

Our study has certain strengths including being the first populationbased estimation of malignant neoplasm of brain incidence that has been conducted in Fars province to this date. Additionally, the data included in this analysis are from high quality registries with a coverage of over $98 \%$. Therefore, the results included should be relatively accurate. Despite Fars being a vast province a limitation would be that no other province in Iran was involved in this study, therefore taking away the opportunity of having access to a larger set of data for our inferences. Finally, it is suggested that future studies investigate the spatio-temporal trend of CNS tumors' mortality in order to determine the extent and severity of this cancer.

\section{Conclusion}

According to geographical and economic conditions, the incidence of CNS tumors is increasing. Changes in lifestyle and the growth of risk factors are the main causes of the increasing incidence of brain tumors in Iran. Therefore, educational programs can be expected to reduce the risk factors and also the complications of CNS tumors by early detection of the tumor.

\section{Funding}

This research did not receive any specific grant from funding agencies in the public, commercial, or not-for-profit sectors.

\section{Declaration of competing interest}

Nothing to declare.

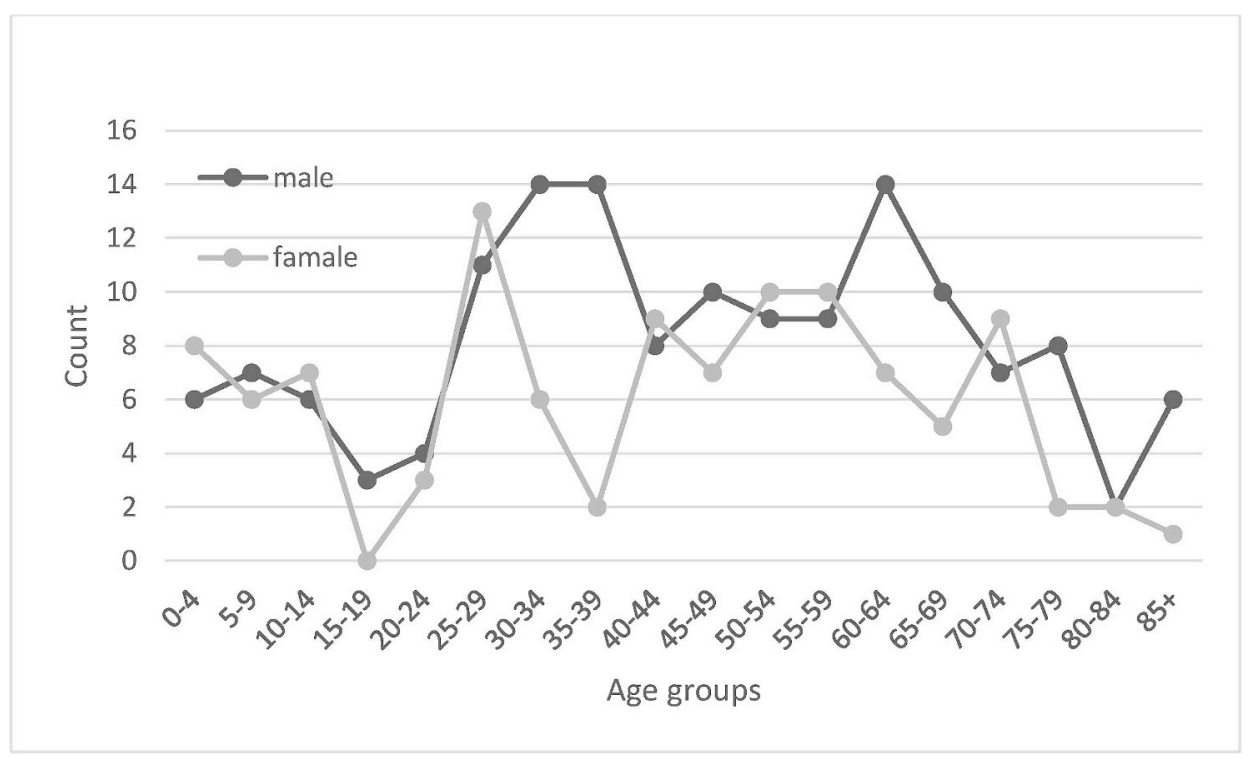

Fig. 4. Age distribution of malignant neoplasm of brain by gender in Fars province at 2015. 


\section{Acknowledgements}

The authors would like to thank the Cancer Research Center of Shiraz University of Medical Sciences for the invaluable coordination and cooperation.

\section{References}

1. de Robles P, Fiest KM, Frolkis AD, et al. The worldwide incidence and prevalence of primary brain tumors: a systematic review and meta-analysis. Neuro Oncol. 2015;17(6):776-783.

2. Askari K, Janeshin S, Mashouf M, Taherzadeh-Amlashi M, Seyed-Saadat S-M. Central nervous system tumors in Guilan, Iran: epidemiological features over 10 years. Casp J Neurol Sci. 2015;1(1):19-26.

3. Hassanipour S, Namvar G, Fathalipour M, Ghorbani M, Abdzadeh E, Zafarshamspour $\mathrm{S}$. The incidence of brain tumours in Iran: a systematic review and meta-analysis. $A d v$ Hum Biol. 2019;9(1):2-7.

4. Manoharan N, Julka PK, Rath GK. Descriptive epidemiology of primary brain and CNS tumors in Delhi, 2003-2007. Asian Pac J Cancer Prev: APJCP. 2012;13(2):637-640.

5. Ambe SN, Lyon KA, Nizamutdinov D, Fonkem E. Incidence trends, rates, and ethnic variations of primary CNS tumors in Texas from 1995 to 2013. Neurooncol Pract. 2018;5(3):154-160.

6. Ferlay J, Shin HR, Bray F, Forman D, Mathers C, Parkin DM. Estimates of worldwide burden of cancer in 2008: GLOBOCAN 2008. Int J Canc. 2010;127(12):2893-2917.

7. Fisher JL, Schwartzbaum JA, Wrensch M, Wiemels JL. Epidemiology of brain tumors. Neurol Clin. 2007;25(4):867-890.

8. Darefsky AS, Dubrow R. International variation in the incidence of adult primary malignant neoplasms of the brain and central nervous system. Cancer Causes Control: CCC. 2009;20(9):1593-1604.

9. Shen F, Wu CX, Yao Y, et al. Transition over 35 years in the incidence rates of primary central nervous system tumors in Shanghai, China and histological subtyping based on a single center experience spanning 60 years. Asian Pac J Cancer Prev: APJCP. 2013;14(12):7385-7393.

10. Pineros M, Sierra MS, Izarzugaza MI, Forman D. Descriptive epidemiology of brain and central nervous system cancers in Central and South America. Canc Epidemiol 2016;44(1) S141-s9.

11. Mehrazin M, Rahmat H, Yavari P. Epidemiology of primary intracranial tumors in Iran, 1978-2003. Asian Pac J Cancer Prev: APJCP. 2006;7(2):283-288.

12. Sadjadi A, Nouraie M, Mohagheghi MA, Mousavi-Jarrahi A, Malekezadeh R, Parkin DM. Cancer occurrence in Iran in 2002, an international perspective. Asian Pac J Cancer Prev: APJCP. 2005;6(3):359-363.

13. Sankoh O, Sharrow D, Herbst K, et al. The INDEPTH standard population for low-and middle-income countries. Glob Health Action. 2013;7(1):23286 2014.

14. Jazayeri SB, Rahimi-Movaghar V, Shokraneh F, Saadat S, Ramezani R. Epidemiology of primary CNS tumors in Iran: a systematic review. Asian Pac J Cancer Prev: APJCP. 2013;14(6):3979-3985

15. Miranda-Filho A, Pineros M, Soerjomataram I, Deltour I, Bray F. Cancers of the brain and CNS: global patterns and trends in incidence. Neuro Oncol. 2017;19(2):270-280.

16. McKinley BP, Michalek AM, Fenstermaker RA, Plunkett RJ. The impact of age and sex on the incidence of glial tumors in New York state from 1976 to 1995. $J$ Neurosurg. 2000;93(6):932-939.

17. Edgren G, Liang L, Adami HO, Chang ET. Enigmatic sex disparities in cancer incidence. Eur J Epidemiol. 2012;27(3):187-196.

18. Dolecek TA, Propp JM, Stroup NE, Kruchko C. CBTRUS statistical report: primary brain and central nervous system tumors diagnosed in the United States in 20052009. Neuro Oncol. 2012;14(Suppl 5):v1-49.

19. Lee CH, Jung KW, Yoo H, Park S, Lee SH. Epidemiology of primary brain and central nervous system tumors in Korea. J Kor Neurosurg Soc. 2010;48(2):145-152.

20. Baldi I, Gruber A, Alioum A, et al. Descriptive epidemiology of CNS tumors in France: results from the Gironde Registry for the period 2000-2007. Neuro Oncol. 2011;13(12):1370-1378.

21. Nilsson J, Holgersson G, Carlsson T, Henriksson R, Bergstrom S, Bergqvist M. Incidence trends in high-grade primary brain tumors in males and females. Oncol Lett. 2017;13(4):2831-2837.

22. Pirouzmand F, Sadanand V. The incidence trends of primary brain tumors in Saskatchewan from 1970 to 2001. Can. J. Neurol. Sci. 2007;34(2):181-186.

23. Wrensch M, Minn Y, Chew T, Bondy M, Berger MS. Epidemiology of primary brain tumors: current concepts and review of the literature. Neuro Oncol. 2002;4(4):278-299.

24. Davis FG, Bruner JM, Surawicz TS. The rationale for standardized registration and reporting of brain and central nervous system tumors in population-based cancer registries. Neuroepidemiology. 1997;16(6):308-316.

25. Nomura E, Ioka A, Tsukuma H. Trends in the incidence of primary intracranial tumors in Osaka, Japan. Jpn J Clin Oncol. 2011;41(2):291-294.

26. Johannesen TB, Angell-Andersen E, Tretli S, Langmark F, Lote K. Trends in incidence of brain and central nervous system tumors in Norway, 1970-1999. Neuroepidemiology. 2004;23(3):101-109.

27. Dobec-Meic B, Pikija S, Cvetko D, et al. Intracranial tumors in adult population of the Varazdin County (Croatia) 1996-2004: a population-based retrospective incidence study. J Neuro-Oncol. 2006;78(3):303-310.

28. Elia-Pasquet S, Provost D, Jaffre A, et al. Incidence of central nervous system tumors in Gironde, France. Neuroepidemiology. 2004;23(3):110-117.

29. Morton LM, Wang SS, Devesa SS, Hartge P, Weisenburger DD, Linet MS. Lymphoma incidence patterns by WHO subtype in the United States, 1992-2001. Blood. 2006;107(1):265-276.

30. Villano JL, Koshy M, Shaikh H, Dolecek TA, McCarthy BJ. Age, gender, and racial differences in incidence and survival in primary CNS lymphoma. Br J Canc. 2011;105(9):1414-1418.

31. Hoffman S, Propp JM, McCarthy BJ. Temporal trends in incidence of primary brain tumors in the United States, 1985-1999. Neuro Oncol. 2006;8(1):27-37. 